\title{
Lactate-enhanced-qSOFA (LqSOFA) score is superior to the other four rapid scoring tools in predicting in-hospital mortality rate of the sepsis patients
}

\author{
Sijia Liu ${ }^{1}$, Chengqi He ${ }^{1}$, Weilue $\mathrm{He}^{2}$, Tian Jiang ${ }^{3}$ \\ ${ }^{1}$ Department of Rehabilitation Medicine, West China Hospital, Sichuan University, Chengdu, China; ${ }^{2}$ Department of Biomedical Engineering, \\ Michigan Technological University, Houghton, Michigan, USA; ${ }^{3}$ Editorial Board of Journal of Sichuan University (Medical Science Edition), \\ Chengdu, China \\ Contributions: (I) Conception and design: S Liu, T Jiang; (II) Administrative support: S Liu, T Jiang; (III) Provision of study materials or patients: All \\ authors; (IV) Collection and assembly of data: All authors; (V) Data analysis and interpretation: S Liu, T Jiang; (VI) Manuscript writing: All authors; \\ (VII) Final approval of manuscript: All authors. \\ Correspondence to: Tian Jiang. Editorial Board of Journal of Sichuan University (Medical Science Edition), Sichuan University, Chengdu, China. \\ Email: 79855467@qq.com.
}

Background: The rising prevalence of early therapy for sepsis has led to the demand for rapid risk-
stratification tools that can estimate the risk of in-hospital mortality for sepsis patients and the need for
intensive care unit (ICU) admission. A robust risk-stratification tool is crucial for in-time sepsis treatment.
This study aimed to compare the abilities of five rapid scoring systems, i.e., LqSOFA score, qSOFA score,
SIRS, MEDS, and MEWS, in predicting the mortality in hospital and ICU admission for sepsis patients.
Methods: A retrospective observational clinical study was conducted in West China Hospital. Our cases
included all patients admitted to the hospital with a diagnosis of sepsis (sepsis-3). We calculated five rapid
prediction scores for the enrolled cases. We then compared each rapid score's ability to predict in-hospital
mortality and ICU admission.

Results: A total of 821 of mixed sepsis patients by sepsis-3 definition were included. The all-cause hospital mortality rate was $21.1 \%$. The LqSOFA score presented the most significant discrimination with an area under the receiver operating characteristic curve (AUC) of 0.751. The AUC of the LqSOFA score for mortality in the hospital was significantly higher than qSOFA (AUC 0.717), SIRS (AUC 0.704), MEDS (AUC 0.670), and MEWS (AUC 0.685).

Conclusions: LqSOFA is a superior prognostic tool for predicting mortality in the hospital. It may provide more exact information for hospital mortality than the other 4 rapid scores in treating sepsis patients.

Keywords: Sepsis; quick Sequential Organ Failure Assessment (qSOFA); prediction; lactate

Submitted Jul 02, 2020. Accepted for publication Aug 06, 2020.

doi: $10.21037 /$ atm-20-5410

View this article at: http://dx.doi.org/10.21037/atm-20-5410

\section{Introduction}

Sepsis is characterized as a "life-threatening organ dysfunction caused by a dysregulated host response to infection" $(1,2)$. Its fatality rate has been reported as up to $24 \%$ in some studies (3-5). Although there have been several iterations of sepsis care bundles in recent years, the core component of all care bundles, such as resuscitation, collection of blood and other specimen, application of broad-spectrum antibiotics, measurement of lactate, intravenous fluids if needed and source control, should be performed without delay to reduce mortality and improve the prognosis of the disease (6). And the timely policy of treatment depends on early and accurate assessment of 
sepsis. Therefore, early and accurate assessments play an essential role in the timely treatment of sepsis. In recent years, the evaluation methods proposed by researchers, such as the sequential organ failure assessment (SOFA) score (1), have high accuracy and widely used. But the SOFA includes laboratory testing parameters, which take time to perform. Therefore, several rapid and simple emergency departments (ED) scoring systems, including quick sequential organ failure assessment (qSOFA) (7), systemic inflammatory response syndrome (SIRS) criterion (8), modified early warning score (MEWS) (9), and mortality in emergency department sepsis (MEDS) (10), have been used to predict the mortality for patients admitted to EDs under various circumstances. Additionally, some recent studies have suggested that the lactate-enhanced-quick sequential organ failure assessment (LqSOFA), which combines the bedside lactate and qSOFA, is a valuable and rapid predicting tool for mortality in sepsis patients $(11,12)$. The study of Amith Shetty and her colleagues (11) enrolled 12,555 cases and showed that the addition of lactate $\geq 2 \mathrm{mmol} / \mathrm{L}$ threshold as an additional point with derivation of the LqSOFA score $\geq 2$ improves the sensitivity in identifying adverse outcomes to $65.5 \%$ in patients with sepsis. Some studies $(13,14)$ have only compared some of these rapid assessment methods, but few research compared the rapid scoring tools with the serum lactate. In 2019, Liu and his colleagues (15) compared the accuracy of the serum lactate, the qSOFA score and the SOFA score for predicting mortality in adults with Sepsis but the author did not assess the performance of LqSOFA, which is a combination of both the lactate and qSOFA. However, the evidence that can directly show the advantages and disadvantages of these rapid tools for assessing sepsis patients is still limited.

Herein, we intend to compare the performance of five evaluative methods of predicting the mortality in sepsis. The hypothesis was that some rapid evaluative methods could present important clinical outcomes more accurately than others in sepsis diagnosis.

We present the following article in accordance with the MDAR reporting checklist (available at http://dx.doi. org/10.21037/atm-20-5410).

\section{Methods}

\section{Study design}

This study used single hospital records of sepsis patient characteristics and the outcomes to compare the performance of five rapid predicting scoring tools.

On patients' records, we calculated each scoring tool, which was then compared with the patient's actual recorded outcome [mortality or intensive care unit (ICU) admission].

For each method, the ROC curve was obtained. And areas under curve (AUC) are calculated to compare the five rapid scoring systems.

The study was conducted in accordance with the Declaration of Helsinki (as revised in 2013) and was approved by the local Institutional Review Committee and the accessed data were anonymized. Because of the retrospective nature of the research, the requirement for informed consent was waived.

\section{Definitions}

The five scores were calculated on the individual dataset at the corresponding sites. The parameters of MEWS are systolic blood pressure, heart rate, respiratory rate, temperature and mental status. MEDS is consists of nine parameters such as age, terminal illness, tachypnea or hypoxia, septic shock, platelet count, band percents, lower respiratory infection, nursing home resident and mental status. SIRS includes four parameters: respiratory rate/ $\mathrm{PaCO}_{2}$, white blood cell count, heart rate, and temperature. The qSOFA includes three parameters: respiratory rate, Glasgow coma score and systolic blood pressure. For the qSOFA score (a range of $0-3$ ), one point was added for serum lactate $\geq 2 \mathrm{mmol} / \mathrm{L}$, so that the LqSOFA scores ranged from 0 to 4 . The details of five scores were showed in Table 1.

\section{Selection of participants}

In this retrospective observational study, we collected raw data from all the cases diagnosed with sepsis in the Emergency Department of our hospital from April 1 $1^{\text {st, }} 2016$ to December 31 2016.

Based on sepsis-3 criteria (1), the diagnostic criteria for sepsis are defined as life-threatening organ dysfunction due to a dysfunctional host response to infection. The organ dysfunction defined as an acute change in total SOFA score $\geq 2$ points cause by the infection.

Inclusion criteria were: (I) age $\geq 18$ years old; (II) diagnosis of sepsis in the medical record. While the exclusion criteria include missing data and pregnant patients.

The treatment strategies of sepsis in our hospital include resuscitation, collection of blood and other samples, source 
Table 1 Sepsis rapid score definitions utilized in our study

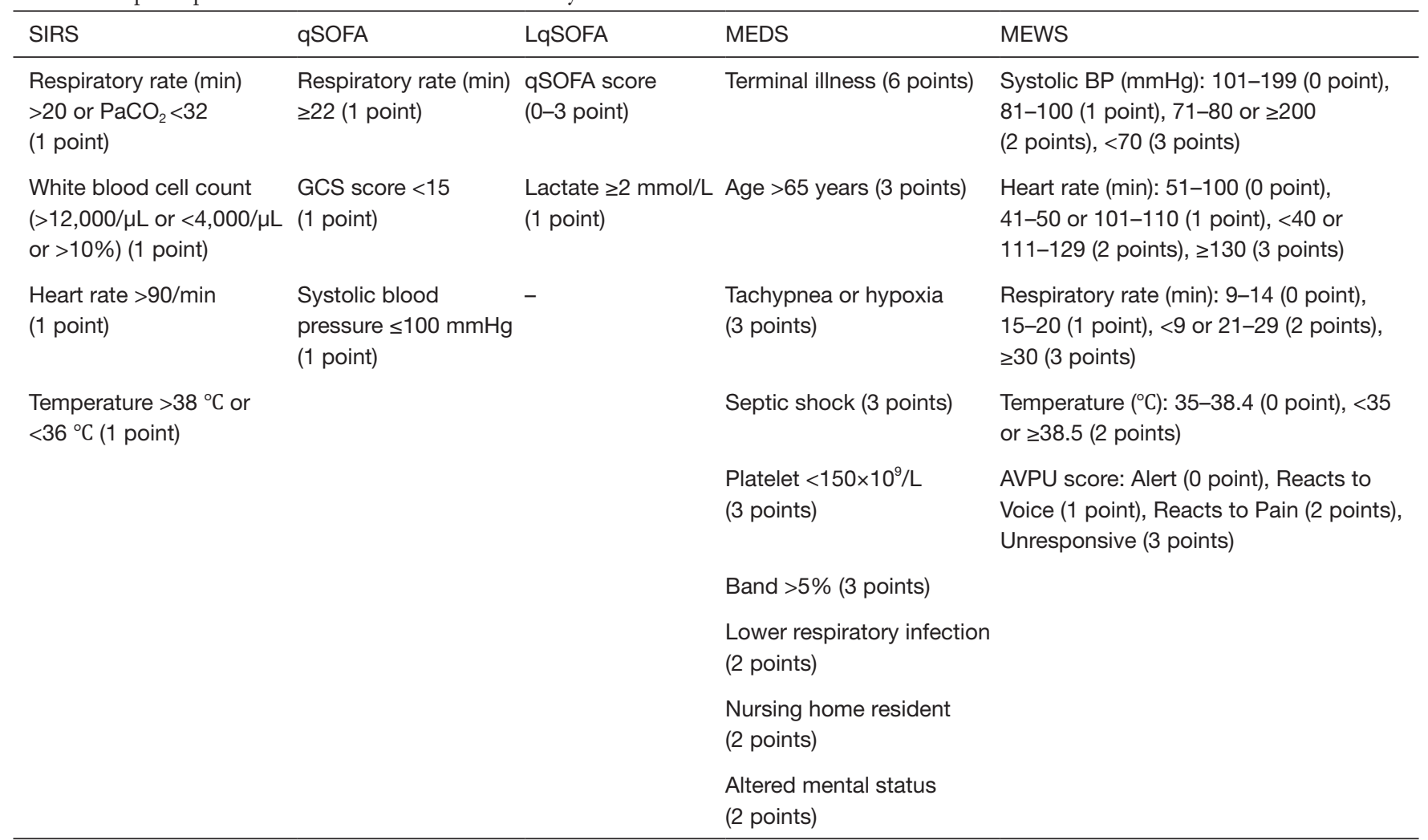

SIRS, systemic inflammatory response syndrome; qSOFA, quick Sequential Organ Failure Assessment; LqSOFA, Lactate enhanced-quick Sequential Organ Failure Assessment; MEDS, mortality in emergency department sepsis; MEWS, modified early warning score.

control, administration of broad-spectrum antibiotics, measurement of lactate, intravenous fluids if hypotensive or raised lactate, and symptomatic treatment.

\section{Data collecting}

In this study, data sources include medical records and death certificates. Before the data collection, the researchers were trained in using the five scoring tools.

There are four components of the collected data:

(I) Baseline characteristics: age, gender, prior major disease (one or more of the following four types of illnesses: diabetes, cancer, cardiovascular disease, and chronic organ dysfunction);

(II) Data of the parameters of the rapid scoring systems (LqSOFA, qSOFA, SIRS, MEWS, and MEDS);

(III) Primary outcome: the hospital mortality for sepsis patients;

(IV) Secondary outcome: ICU admission of sepsis patients during hospitalization.

\section{Statistical analysis}

The statistical description of normal distribution measurement data is expressed as mean \pm standard deviation. While the non-normally distributed measurement data is expressed in medians and quartiles. Further, the statistical description of the enumeration data is represented by the constituent ratio. And the Hanley and McNeil method, which performed by MedCalc software, was used for estimating the sample size.

The primary statistical analysis was applied to the receiver operator curves (ROC) with associated area under curve (AUC). All these analyses were performed with SPSS software version 17 (SPSS, Inc.) and MedCalc software ver. 12.7.0.0.

\section{Results}

In this study, 821 patient records were enrolled, which were from 865 records in total. A breakdown of how the data were obtained from the hospital records is illustrated in Figure 1. 


\section{Baseline characteristics}

In our study, the mean age of the enrolled cases was $58.3 \pm 17.09$ years. Among the cases, $528(64.3 \%)$ were males and $173(21.1 \%)$ patients died in the hospital. And the details of the baseline data are listed in Table 2 .

\section{Comparison of the scoring system}

With hospital mortality as the standard for comparison, the order of the AUC from high to low was LqSOFA (0.751),

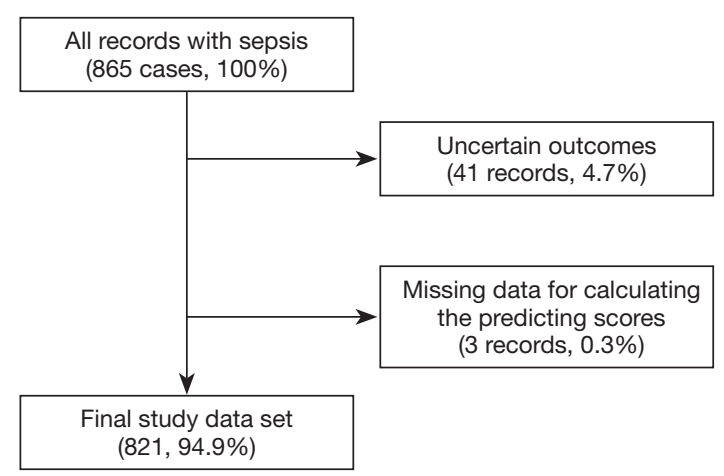

Figure 1 The processes of enrolling cases from source records to obtain the dataset of interest in this study.
qSOFA (0.717), SIRS (0.704), MEWS (0.685) and MEDS (0.670) (Figure 2 and Table 3). Among them, LqSOFA was superior to the other four rapid scoring systems $(\mathrm{P}<0.05$, Table 4). And there was no significant difference between any two of the other scoring systems (qSOFA, MEDS, SIRS, and MEWS).

With the ICU admission as the standard, the descending order of AUC was SIRS 0.701, LqSOFA 0.687, qSOFA 0.676 , MEWS 0.671 , and MEDS 0.662 (Figure 3 and Table 5). However, there was no significant difference between any two of the scoring systems (Table 6).

\section{Discussion}

Screening tests aim at discovering latent risks from the well. Rapid scores and classification systems for sepsis, however, support decision-making, and management. They enable the clinicians to stratify the risk of adverse outcomes in sepsis patients (11). Selective screening of high-risk groups in hospitals may be used to select patients requiring more complex investigations and management. Flagging patients with an elevated risk of mortality in sepsis can give rise to a more efficient construct of therapeutic strategies, including investigation, monitoring, and intense therapies. Predicting tools with high sensitivity and low specificity can

Table 2 Baseline characteristics

\begin{tabular}{|c|c|c|c|c|c|}
\hline Parameters & Mean & Standard deviation & Median & $25 \%$ quartile & $75 \%$ quartile \\
\hline Temperature $\left({ }^{\circ} \mathrm{C}\right)$ & 37.2 & 1.26 & - & - & - \\
\hline Heart rate $(\mathrm{bpm})$ & 109.2 & 20.33 & - & - & - \\
\hline Respiratory rate (bpm) & 23.6 & 5.15 & - & - & - \\
\hline Diastolic pressure $(\mathrm{mmHg})$ & 73 & 16.83 & - & - & - \\
\hline $\mathrm{SPO}_{2}(\%)$ & 93.7 & 7.43 & - & - & - \\
\hline SOFA & - & - & 5 & 3 & 8 \\
\hline LqSOFA & - & - & 1 & 1 & 2 \\
\hline MEWS & - & - & 4 & 2 & 5 \\
\hline SIRS & - & - & 9 & 8 & 12 \\
\hline
\end{tabular}

SIRS, systemic inflammatory response syndrome; qSOFA, quick Sequential Organ Failure Assessment; LqSOFA, Lactate enhanced-quick Sequential Organ Failure Assessment; MEDS, mortality in emergency department sepsis; MEWS, modified early warning score. 


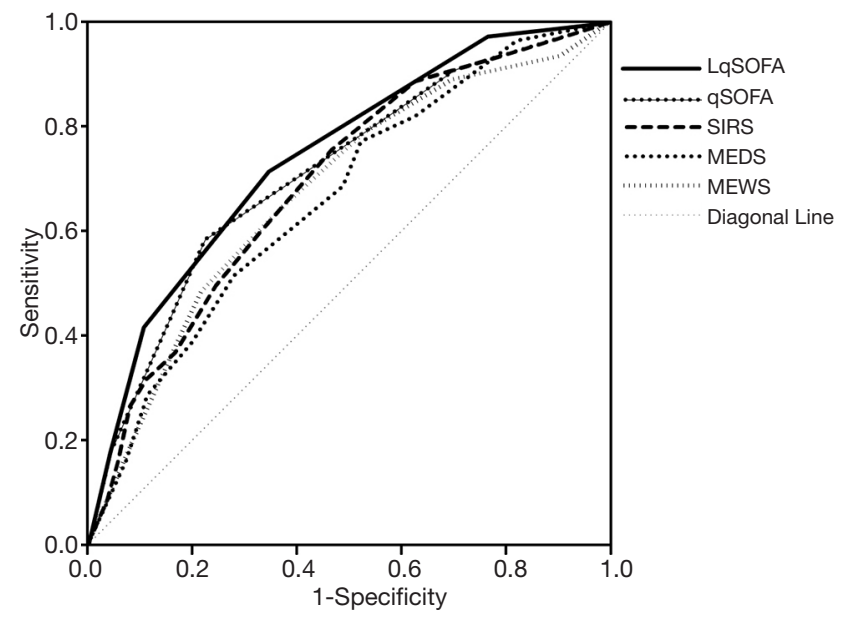

Figure 2 ROC curves for comparing the scoring systems with inhospital mortality. LqSOFA, Lactate enhanced-quick Sequential Organ Failure Assessment; qSOFA, quick Sequential Organ Failure Assessment; SIRS, systemic inflammatory response syndrome; MEDS, mortality in emergency department sepsis; MEWS, modified early warning score.

Table 3 Comparison of the scoring system with in-hospital mortality

\begin{tabular}{lccc}
\hline Scoring systems & AUC & SE & $95 \% \mathrm{Cl}$ \\
\hline LqSOFA & 0.751 & 0.0287 & 0.720 to 0.780 \\
qSOFA & 0.717 & 0.0312 & 0.685 to 0.748 \\
SIRS & 0.704 & 0.0298 & 0.672 to 0.735 \\
MEWS & 0.685 & 0.0321 & 0.652 to 0.717 \\
MEDS & 0.670 & 0.0314 & 0.636 to 0.702 \\
\hline
\end{tabular}

95\% Cl, 95\% confidence interval; SE, standard error; AUC, area under the curve; SIRS, systemic inflammatory response syndrome; qSOFA, quick Sequential Organ Failure Assessment; LqSOFA, Lactate enhanced-quick Sequential Organ Failure Assessment; MEDS, mortality in emergency department sepsis; MEWS, modified early warning score.

lead to inappropriate allocation of resources, while tools with low sensitivity can lead to insufficient trust. Previous studies have suggested that early assessment has significant implications for the timely treatment and management of sepsis, which could reduce the risk of death for critical sepsis patients in the emergency department $(16,17)$.

This study compared the discriminatory ability of LqSOFA, qSOFA, SIRS, MEWS, and MEDS for evaluating sepsis patients. Among these five screening tools,
MEDS and MEWS have the most number of indicators and are relatively complex. SIRS has fewer parameters than the former two but its accuracy is considered to be slightly lower than qSOFA (2). The qSOFA is simple and recommended in the sepsis-3 criteria (1), but recently studies still questioned its accuracy $(18,19)$. The serum lactate level required by the LqSOFA can be completed by point-of-care testing, so the LqSOFA can be used as bedside tools for screening sepsis. But the predicting value of the LqSOFA needs further verification due to the short application time of the LqSOFA.

The SIRS criteria have been used to assess sepsis since over two decades ago. However, SIRS criteria were found to be insufficiently specific to assess the mortality of sepsis patients. Kaukonen KM's study (20) has shown that SIRS criteria fail to define a transition point in the risk of death and may not be a perfect predictor of mortality. The qSOFA score $(21,22)$ has been proposed as a risk stratification tool. It is more specific than the SIRS criteria and can more efficiently urge the assessment of in-hospital mortality, initiate appropriate sepsis therapy, refer patients to the ICU, and help identify life-threatening sepsis.

The qSOFA recommendation was initially formed from the retrospective analysis of a database, and the debate on its clinical usefulness soon appeared (23-28). Recently, Churpek and colleagues (29) found that the qSOFA performed no more effective than the MEWS in predicting in-hospital mortality and ICU transfer in sepsis patients. Our study found reduced sensitivity of the qSOFA score, like the recent prospective evaluations. In our study, there was no significant difference in the ability to evaluate in-hospital mortality in sepsis among the qSOFA, SIRS, MEWS, and MEDS.

Lately, some studies $(11,12)$ have suggested that combining the bedside lactate and qSOFA score can be a valuable method for predicting the mortality for sepsis. In a study of 12,555 patients from multiple EDs in Australia and the Netherlands, post hoc addition of lactate $\geq 2 \mathrm{mmol} / \mathrm{L}$ cut-off to the qSOFA score resulted in an improved sensitivity for identifying patients at risk of mortality. Amith Shetty and her colleagues named the combined tool as LqSOFA, an effective predictor of mortality in sepsis. They showed that in-hospital mortality was related to LqSOFA scores in the patients who were critically ill with an AUROC of 0.74. LqSOFA showed significantly greater capability compared with qSOFA are suggested for screening by the sepsis-3 criteria. Our study illustrated that these findings are consistent with these reports.

Also, we found that these scores were less exact for 
Table 4 Pairwise comparison of ROC curves with in-hospital mortality

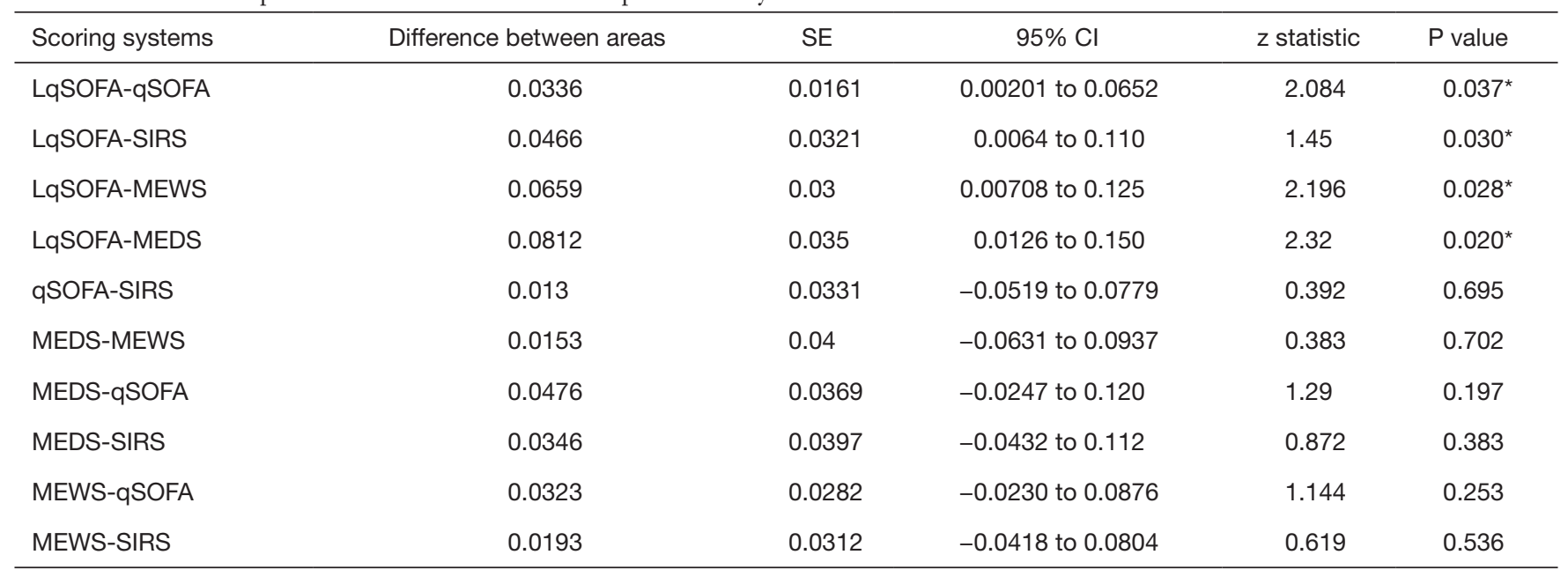

*, $\mathrm{P}<0.05 .95 \% \mathrm{Cl}, 95 \%$ confidence interval; SE, standard error; SIRS, systemic inflammatory response syndrome; qSOFA, quick Sequential Organ Failure Assessment; LqSOFA, Lactate enhanced-quick Sequential Organ Failure Assessment; MEDS, mortality in emergency department sepsis; MEWS, modified early warning score.

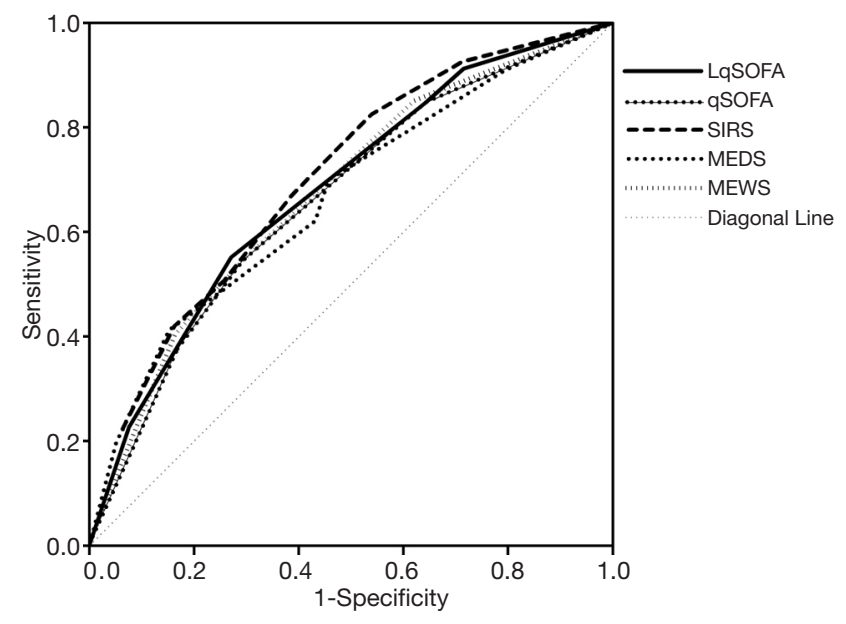

Figure 3 ROC curves obtained from the predicting tools with ICU admission. ICU, intensive care unit; LqSOFA, Lactate enhanced-quick Sequential Organ Failure Assessment; qSOFA, quick Sequential Organ Failure Assessment; SIRS, systemic inflammatory response syndrome; MEDS, mortality in emergency department sepsis; MEWS, modified early warning score.

early assessment of ICU admission. Further studies should be carried out for early assessment of ICU admission, to achieve rapid and effective ICU resource allocation for critical sepsis patients, especially when the emergency department is overcrowded (6).

However, the limitations of our pilot study were its
Table 5 Comparison of the scoring systems with ICU admission

\begin{tabular}{|c|c|c|c|}
\hline Scoring systems & $A \cup C$ & SE & $95 \% \mathrm{Cl}$ \\
\hline LqSOFA & 0.687 & 0.0188 & 0.654 to 0.718 \\
\hline qSOFA & 0.676 & 0.0191 & 0.643 to 0.708 \\
\hline SIRS & 0.701 & 0.0185 & 0.669 to 0.733 \\
\hline MEWS & 0.671 & 0.0192 & 0.637 to 0.703 \\
\hline MEDS & 0.662 & 0.0195 & 0.629 to 0.695 \\
\hline \multicolumn{4}{|c|}{$\begin{array}{l}95 \% \mathrm{CI}, 95 \% \text { confidence interval; SE, standard error; AUC, } \\
\text { area under curve; ICU, intensive care unit; SIRS, systemic } \\
\text { inflammatory response syndrome; qSOFA, quick Sequential } \\
\text { Organ Failure Assessment; LqSOFA, Lactate enhanced-quick } \\
\text { Sequential Organ Failure Assessment; MEDS, mortality in } \\
\text { emergency department sepsis; MEWS, modified early warning } \\
\text { score. }\end{array}$} \\
\hline
\end{tabular}

retrospective study design, insufficient sample size, and missing data. The subgroup of septic shock was not analyzed due to its small sample size. Our ongoing study is about the prospective multiple center studies with adjustments for the effectiveness and application prospect of early evaluative scoring systems for sepsis.

\section{Conclusions}

The LqSOFA score can predict in-hospital mortality in sepsis more accurately than qSOFA, SIRS, MEWS, and 
Table 6 Pairwise comparison of ROC curves with ICU admission

\begin{tabular}{|c|c|c|c|c|c|}
\hline Scoring systems & Difference between areas & SE & $95 \% \mathrm{Cl}$ & z statistic & $P$ value \\
\hline LqSOFA-MEWS & 0.0158 & 0.0202 & -0.0238 to 0.0555 & 0.781 & 0.435 \\
\hline LqSOFA-qSOFA & 0.0104 & 0.0109 & -0.0109 to 0.0317 & 0.959 & 0.338 \\
\hline LqSOFA-SIRS & 0.0147 & 0.0215 & -0.0274 to 0.0569 & 0.686 & 0.493 \\
\hline MEWS-qSOFA & 0.00538 & 0.0189 & -0.0317 to 0.0425 & 0.284 & 0.776 \\
\hline MEDS-qSOFA & 0.0141 & 0.023 & -0.0309 to 0.0591 & 0.613 & 0.540 \\
\hline MEDS-SIRS & 0.0392 & 0.0247 & -0.00921 to 0.0877 & 1.587 & 0.112 \\
\hline MEWS-SIRS & 0.0306 & 0.0193 & -0.00734 to 0.0684 & 1.58 & 0.114 \\
\hline
\end{tabular}

95\% Cl: 95\% confidence interval; SE, standard error; ICU, intensive care unit; SIRS, systemic inflammatory response syndrome; qSOFA, quick Sequential Organ Failure Assessment; LqSOFA, Lactate enhanced-quick Sequential Organ Failure Assessment; MEDS, mortality in emergency department sepsis; MEWS, modified early warning score.

MEDS. At sites where qSOFA score is being considered, and bedside lactate examination is readily available, the LqSOFA score rather than qSOFA alone should be prospectively confirmed.

\section{Acknowledgments}

Funding: This work was supported by the Research Fund of Natural Science Foundation of China (NSFC) (No. 81572236), the National Health and Family Planning Commission of China (No. 201302003), the Health and Family Planning Commission of Chengdu City (No. CDWSYJ-2016-01), and the Chengdu Science and Technology Bureau (No. 2015-HM02-00042-SF), Sichuan Science and Technology Department Technology - Key R\&D Project (No. 2019YFS0150).

\section{Footnote}

Reporting Checklist: The authors have completed the MDAR reporting checklist. Available at http://dx.doi.org/10.21037/ atm-20-5410

Data Sharing Statement: Available at http://dx.doi. org/10.21037/atm-20-5410

Conflicts of Interest: All authors have completed the ICMJE uniform disclosure form (available at http://dx.doi. org/10.21037/atm-20-5410). The authors have no conflicts of interest to declare.

Ethical Statement: The authors are accountable for all aspects of the work in ensuring that questions related to the accuracy or integrity of any part of the work are appropriately investigated and resolved. The study was conducted in accordance with the Declaration of Helsinki (as revised in 2013) and was approved by the local Institutional Review Committee and the accessed data were anonymized. Because of the retrospective nature of the research, the requirement for informed consent was waived.

Open Access Statement: This is an Open Access article distributed in accordance with the Creative Commons Attribution-NonCommercial-NoDerivs 4.0 International License (CC BY-NC-ND 4.0), which permits the noncommercial replication and distribution of the article with the strict proviso that no changes or edits are made and the original work is properly cited (including links to both the formal publication through the relevant DOI and the license). See: https://creativecommons.org/licenses/by-nc-nd/4.0/.

\section{References}

1. Singer M, Deutschman CS, Seymour CW, et al. The Third International Consensus Definitions for Sepsis and Septic Shock (Sepsis-3). JAMA 2016;315:801-10. 
2. Haydar S, Spanier M, Weems P, et al. Comparison of QSOFA score and SIRS criteria as screening mechanisms for emergency department sepsis. Am J Emerg Med 2017;35:1730-3.

3. Schrijver IT, Kemperman H, Roest M, et al. Myeloperoxidase can differentiate between sepsis and noninfectious SIRS and predicts mortality in intensive care patients with SIRS. Intensive Care Med Exp 2017;5:43.

4. Brooks D, Polubothu P, Young D, et al. Sepsis caused by bloodstream infection in patients in the intensive care unit: the impact of inactive empiric antimicrobial therapy on outcome. J Hosp Infect 2018;98:369-74.

5. Uhel F, Peters-Sengers H, van der Poll T. Initiation of renal replacement therapy in patients with sepsis: more to it than meets the eye. Ann Transl Med 2018;6:S130.

6. Hunt A. Sepsis: an overview of the signs, symptoms, diagnosis, treatment and pathophysiology. Emerg Nurse 2019;27:32-41.

7. $\mathrm{Hu} \mathrm{H}, \mathrm{Du} \mathrm{X}, \mathrm{He} \mathrm{Y}$, et al. Application of SOFA score as a predict tool for mortality of delayed admission to ICU on patients with acute respiratory failure. Am J Emerg Med 2017;35:914-5.

8. Rello J, Rubulotta F. Best practice for sepsis. J Thorac Dis 2018;10:1280-2.

9. Tirotta D, Gambacorta M, La Regina M .Evaluation of the threshold value for the modified early warning score (MEWS) in medical septic patients: a secondary analysis of an Italian multicentric prospective cohort (SNOOPII study). QJM 2017;110:369-73.

10. Macdonald SP, Arendts G, Fatovich DM. Comparison of PIRO, SOFA, and MEDS scores for predicting mortality in emergency department patients with severe sepsis and septic shock. Acad Emerg Med 2014;21:1257-63.

11. Shetty A, MacDonald SP, Williams JM, et al. Lactate $\geq 2 \mathrm{mmol} / \mathrm{L}$ plus qSOFA improves utility over qSOFA alone in emergency department patients presenting with suspected sepsis. Emerg Med Australas 2017;29:626-34.

12. Du XM, Hu H, Kurbah OM. Both qSOFA score and bedside plasma lactate are the predictors of mortality for patients with infections in ED. Am J Emerg Med 2017;35:1381-2.

13. Feist B. Screening for sepsis: SIRS or qSOFA? A literature review. Emerg Nurse 2019;27:13-8.

14. van der Woude SW, van Doormaal FF, Hutten BA, J Nellen F, Holleman F. Classifying sepsis patients in the emergency department using SIRS, qSOFA or MEWS.
Neth J Med 2018;76:158-66.

15. Liu Z, Meng Z, Li Y, et al. Prognostic accuracy of the serum lactate level, the SOFA score and the qSOFA score for mortality among adults with Sepsis. Scand J Trauma Resusc Emerg Med 2019;27:51.

16. Chen CC, Chong CF, Liu YL, et al. Risk stratification of severe sepsis patients in the emergency department. Emerg Med J 2006;23:281-5.

17. Jones AE, Saak K, Kline JA. Performance of the Mortality in emergency department Sepsis score for predicting hospital mortality among patients with severe sepsis and septic shock. Am J Emerg Med 2008;26:689-92.

18. Dorsett M, Kroll M, Smith CS, et al. qSOFA Has Poor Sensitivity for Prehospital Identification of Severe Sepsis and Septic Shock. Prehosp Emerg Care 2017;21:489-97.

19. Askim $\AA$, Moser F, Gustad LT, et al. Poor performance of quick-SOFA (qSOFA) score in predicting severe sepsis and mortality - a prospective study of patients admitted with infection to the emergency department. Scand J Trauma Resusc Emerg Med 2017;25:56.

20. Kaukonen KM, Bailey M, Bellomo R. Systemic Inflammatory Response Syndrome Criteria for Severe Sepsis. N Engl J Med 2015;373:881.

21. Du B, Weng L. Systemic inflammatory response syndrome, sequential organ failure assessment, and quick sequential organ failure assessment: more pieces needed in the sepsis puzzle. J Thorac Dis 2017;9:452-4.

22. Finkelsztein EJ, Jones DS, Ma KC, et al. Comparison of qSOFA and SIRS for predicting adverse outcomes of patients with suspicion of sepsis outside the intensive care unit.Crit Care 2017;21:73.

23. GunesOzaydin M, Guneysel O, Saridogan F, et al. Are scoring systems sufficient for predicting mortality due to sepsis in the emergency department? Turk J Emerg Med 2016;17:25-8.

24. Umemura Y, Ogura H, Gando S, et al. Assessment of mortality by qSOFA in patients with sepsis outside ICU: A post hoc subgroup analysis by the Japanese Association for Acute Medicine Sepsis Registry Study Group. J Infect Chemother 2017;23:757-62.

25. Hou PC, Seethala RR, AisikuIP.qSOFA—welcome to the sepsis alphabet soup. J Thorac Dis 2017;9:965-8.

26. Vincent JL, Grimaldi D. Quick sequential organ failure assessment: big databases vs. intelligent doctors. J Thorac Dis 2016;8:E996-8.

27. Raith EP, Udy AA, Bailey M, et al. Prognostic accuracy of the SOFA Score, SIRS Criteria, and qSOFA Score 
for in-hospital mortality among adults with suspected infection admitted to the intensive care unit. JAMA 2017;317:290-300.

28. Huang YL, Min J, Li GH, et al. Chronic obstructive pulmonary disease Comorbidity Clinical study Systemic inflammatory response. Journal of Sichuan University (medical science edition) 2019;50:88-92.

Cite this article as: Liu S, He C, He W, Jiang T. Lactateenhanced-qSOFA (LqSOFA) score is superior to the other four rapid scoring tools in predicting in-hospital mortality rate of the sepsis patients. Ann Transl Med 2020;8(16):1013. doi: $10.21037 /$ atm-20-5410
29. Churpek MM, Snyder A, Han X, et al. Quick Sepsisrelated organ failure assessment, systemic inflammatory response syndrome, and early warning scores for detecting clinical deterioration in infected patients outside the intensive care unit. Am J Respir Crit Care Med 2017;195:906-11. 